\title{
DIAGNÓSTICO SITUACIONAL DE LA CRIANZA DE CUYES EN UNA ZONA DE CAJAMARCA
}

\author{
Situational Diagnostic of the Breeding of Guinea Pigs in Cajamarca
}

Gisel Aguilar R. ${ }^{1}$, José Bustamante L. ${ }^{1,2}$, Víctor Bazán R. ${ }^{3}$, Néstor Falcón P. ${ }^{4}$

\section{RESUMEN}

Se realizó una encuesta en julio de 2004 a 160 familias criadoras de cuyes en el distrito de Santa Cruz, Cajamarca, con el objetivo de caracterizar los sistemas de producción de cuyes. El 44.6\% de los productores tenía más de 50 años de edad y el $42 \%$ de las esposas tenía entre 31 a 50 años. Primaria era el grado de instrucción predominante. La ocupación del jefe de familia era la agricultura (95.4\%) y de la esposa era su casa (97.2\%). La crianza de cuyes era conducida por el ama de casa bajo un sistema familiar o tradicional. Se encontró un promedio de 20.4 cuyes por familia, criados en un solo grupo sin distinción de clase, sexo y edad, de preferencia en la cocina (88.8\%), donde permanecían sueltos (73.8\%) o en pozas (21.9\%). La alimentación se basó en forrajes, malezas y residuos de cocina. Las principales enfermedades reportadas fueron ectoparásitos (90.1\%) y la "peste” (76\%). El 71.2\% de las familias destinaba los cuyes para autoconsumo y venta, y el 28.2\% sólo para autoconsumo. El 96.2\% de los encuestados no disponía de servicios de asistencia técnica, créditos, insumos, etc. El 67.5\% de los criadores consideraba que mejorarían su crianza con asistencia técnica.

Palabras clave: cuyes, encuestas, caracterización, sistemas de producción, métodos de crianza

\section{Abstract}

A survey was conducted in July 2004 to 160 families rearing Guinea pigs in the district of Santa Cruz, Cajamarca, with the aim of characterizing the production system of these animals. Men were more than 50 years of age (44.6\%) and wives were mainly between 31 to 50 years old (42\%). Primary school was the main grade of instruction. The main labour activity of the head of the family was agriculture (95.4\%) and housekeeping for women (97.2\%). Raising guinea pigs was mainly driven by the housewife under a family or traditional system. There were 20.4 guinea pigs on average per family, reared regardless of class, sex and age, preferably in the kitchen (88.8\%), free (73.8\%) or in pools (21.9\%). Feeding was based on forages, weeds and kitchen waste. The main diseases reported were ectoparasites (90.1\%) and the «plague» (76\%). Guinea pigs were used for

\footnotetext{
${ }^{1}$ Laboratorio de Producción Avícola y Especies Menores, ${ }^{3}$ Laboratorio de Zootecnia y Producción Agropecuaria, Facultad de Medicina Veterinaria, Universidad Nacional Mayor de San Marcos, Lima

${ }^{2}$ E-mail: jabustamantel1@yahoo.es

${ }^{4}$ Facultad de Veterinaria y Zootecnia, Universidad Peruana Cayetano Heredia, Lima
} 
consumption and sale (71.2\%) or only for home consumption (28.2\%). Most of producers (96.2\%) indicated that they do not have services such as technical assistance, credit, inputs, etc, and $67.5 \%$ considered that they would improve their productivity if technical assistance was available.

Key words: guinea pigs, surveys, characterization, production systems, breeding methods

\section{INTRODUCCIÓN}

Todo sistema de producción agropecuaria es una combinación de factores y procesos que actúan como un todo y que interactúan entre sí (Ruíz, 1989). Los sistemas de producción pecuaria están supeditados a la ecología, grado de tecnificación, ubicación geográfica, estrato socioeconómico, disponibilidad de servicios, tipo de comercialización y tenencia de tierras (Kajjak, 1995); por lo que se hace necesario recurrir a la caracterización de sus componentes a fin de buscar la forma de hacerlos más eficientes dentro del sistema (Zaldívar, 1989), y receptivos de una implementación de servicios técnicos, factibles de ser adoptados por el productor.

La caracterización es la etapa central del análisis de sistemas. Para ello, se utiliza información secundaria, sondeos, encuestas de una sola visita (diagnóstico situacional o estático) y de varias visitas a través de un ciclo productivo (diagnóstico dinámico) (Ruíz, 1989). Los sistemas de producción de cuyes identificados y caracterizados en el Perú son familiar, familiar-comercial y comercial (Chauca et al., 1994).

En el Perú, el departamento de Cajamarca posee la mayor población de cuyes (INEI, 2004b), la cual representa una zona potencial para la crianza tecnificada de esta especie. Si bien se han realizado estudios sobre la producción de cuyes en la zona, se conoce poco sobre su situación actual. El propósito del presente estudio fue caracterizar los sistemas de producción de cuyes existentes en el distrito de Santa Cruz, identificando los factores limitantes y la demanda tecnológica.

\section{MATERIALES y MÉTOdos}

El distrito de Santa Cruz, capital de la provincia del mismo nombre, está situado a $210 \mathrm{~km}$ al noroeste de la ciudad de Cajamarca, a una altitud de $2035 \mathrm{msnm}$. La zona rural está dividida en 26 caseríos y 23 anexos (INEI, 2004a).

El estudio se llevó a cabo en julio de 2004 en los centros poblados de 11 caseríos, constituyendo el $42.3 \%$ del total de caseríos del distrito. Los caseríos se eligieron al azar tomando como referencia la clasificación por microcuencas de la Agencia Santa Cruz del Programa Nacional de Manejo de Cuencas Hidrográficas y Conservación de Suelos (PRONAMACHS), que divide el territorio distrital en tres zonas (Cuadro 1).

Se utilizó parte de la metodología de investigación recomendada en el Enfoque de Sistemas Agropecuarios (Ruíz, 1989). A partir de entrevistas a autoridades locales y un sondeo, se determinó el número aproximado de familias criadoras de cuyes por caserío.

Se desarrollaron encuestas de una sola visita. Las familias encuestadas fueron escogidas al azar, alcanzando una cobertura mínima del 50\% de las familias criadoras de cuyes, a excepción del caserío de Maraypampa donde se consideró el 20\% por ser el más poblado. El número total de encuestas fue de 160 (Cuadro 1). 
Cuadro 1. Caseríos del distrito de Santa Cruz seleccionados para una encuesta de una sola visita a fin de caracterizar el sistema de producción de cuyes

\begin{tabular}{|c|c|c|c|c|c|}
\hline \multirow{3}{*}{ Microcuenca $^{1}$} & \multirow{3}{*}{ Caserío } & \multirow{3}{*}{$\begin{array}{c}\text { Viviendas } \\
\left(\mathrm{N}^{0}\right)^{2}\end{array}$} & \multicolumn{3}{|c|}{ Familias criadoras de cuyes ${ }^{3}$} \\
\hline & & & \multirow{2}{*}{$\mathrm{N}^{\mathrm{o}}$} & \multicolumn{2}{|c|}{ Familias encuestadas } \\
\hline & & & & $\mathrm{N}^{\circ}$ & $\%$ \\
\hline \multirow{3}{*}{ Cañad } & Atumpampa & 47 & 28 & 14 & 50.0 \\
\hline & Barriada Nueva & 20 & 10 & 10 & 100.0 \\
\hline & Maraypampa & 220 & 170 & 34 & 20.0 \\
\hline \multirow{4}{*}{ El Chileno } & Chambac & 70 & 40 & 20 & 50.0 \\
\hline & El Quio & 30 & 15 & 10 & 66.7 \\
\hline & La Lamud & 15 & 10 & 10 & 100.0 \\
\hline & Mayobamba & 50 & 30 & 15 & 50.0 \\
\hline \multirow{4}{*}{ Las Nieves } & La Cárcel & 35 & 20 & 10 & 50.0 \\
\hline & Mitopampa & 50 & 27 & 15 & 55.6 \\
\hline & Santa Rita & 40 & 24 & 12 & 50.0 \\
\hline & Tambuden & 40 & 20 & 10 & 50.0 \\
\hline Total & 11 caseríos & 597 & 394 & 160 & 40.6 \\
\hline
\end{tabular}

Los resultados de las encuestas fueron registrados en una base de datos, elaborada a través del programa de cómputo Visual Basic.Net. A partir de esta base de datos y con el apoyo del programa de cómputo SQL Server 2000, se clasificó y tabuló la información. Los resultados son presentados por medio de una estadística descriptiva.

\section{Resultados y Discusión}

\section{Productor}

En el caso de los hombres, el $44.6 \%$ supera los 50 años de edad, en tanto que el rango de edad predominante de las esposas fue de 31 a 50 años (42.1\%). La mayoría de los hijos que viven con los padres (49.0\%) tenían entre 6 a 17 años de edad. El grado de instrucción, en la mayoría de miembros de la familia (65.6\%), era primaria completa o incompleta. La ocupación principal del jefe de familia era la agricultura (95.4\%) y de la esposa era su casa (97.2\%).

\section{Infraestructura}

El acceso directo a la mayoría de viviendas era a través de trochas no carrozables. Las viviendas eran de pobre condición y carentes de servicios básicos, construidas principalmente de adobe y quincha. Sólo el caserío de Chambac y parte del caserío de Mayobamba contaban con fluido eléctrico. El agua provenía de piletas instaladas fuera de las viviendas, pozas o puquiales. 


\section{Componente Agrícola}

La tenencia de tierras se caracterizaba por ser minifundista, con una extensión promedio de 2.31 ha por unidad agropecuaria, predominando el cultivo alimenticio o de pan llevar. En los terrenos mixtos, cerca del 10\% del área estaba destinada a cultivos forrajeros.

\section{Crianza Pecuaria}

El tipo de crianza era familiar, con escasas técnicas de manejo y destinado principalmente al autoconsumo. El 96.3\% de las familias criaban aves de corral, el 64.4\% porcinos, el $63.1 \%$ bovinos, el $41.9 \%$ equinos y $13.1 \%$ ovinos. El rango era de 1 a 5 animales por especie. En la crianza de cuyes, predominaban los núcleos de 11 a 20 animales (34.4\%), con un promedio de 20.4 cuyes por familia; promedio similar al encontrado en otras zonas de Cajamarca, donde el hato familiar de cuyes consta en promedio de 25.6 unidades y en otras partes de la Sierra Central es de 20.5 cuyes (Chauca et al., 1994).

\section{Caracterización de la Crianza}

La crianza de cuyes está considerada como parte de las labores domésticas, siendo desempeñada principalmente por el ama de casa.

\section{Tipos de cuyes}

El tipo predominante según su conformación era del tipo B (58.3\%). De estos, el $62.8 \%$ era de pelaje tipo 1 y el $36.7 \%$ de tipo 2. Según Chauca (1997), el tipo B es el que caracteriza al cuy criollo, donde existe una predominancia de los tipos 1 (60.65\%) y 2 (33.32\%).

\section{Composición del plantel}

Se registró un alto porcentaje de cuyes reproductores (44.8\%), aunque se tiene registros de $54.4 \%$ en la Costa Central y $52.0 \%$ en la Sierra Norte (Chauca et al., 1994). Se requiere mejorar la eficiencia productiva con el fin de reducir estos valores a un tercio de la población (Chauca, 1997). El número de cuyes lactantes fue solamente del $15.9 \%$, debido aparentemente a la alta mortalidad de crías. Esta mortalidad en crianzas familiares puede alcanzar el 38\% (Chauca, 1997).

\section{Alimentación}

La alimentación fue a base de forrajes, malezas y residuos de cocina y de cosecha. Los principales forrajes y malezas fueron panca de maíz (88.8\%), caña de azúcar (50.0\%), nudillo (47.5\%), kikuyo (46.9\%), maicillo (34.9\%), y hoja de plátano (34.4\%). Apenas el 2.5\% de encuestados manifestó utilizar alfalfa, a diferencia de otras zonas de Cajamarca donde la alfalfa (34.7\%), el rye grass $(20.8 \%)$ y la retama (17.3\%) tienen especial importancia (Chauca et al., 1994).

\section{Instalaciones}

Los cuyes eran criados en un solo grupo, sin distinción de clase, sexo y edad en todos los casos, y principalmente en la cocina $(88.8 \%)$ o en otros ambientes como el dormitorio, almacén o galpón (11.2\%), donde permanecen sueltos (73.8\%) e inclusive junto a otros animales. Este tipo de alojamiento y manejo es encontrado en el $90 \%$ de las fincas registradas en el proyecto "Sistemas de Producción de Cuyes en el Perú” (Chauca et al., 1994). El 21.9\% de los encuestados utilizaba de 1 a 2 pozas, el 3.1\% empleaba corrales y un $1.2 \%$ los tenía en jaulas. Los materiales utilizados eran los más económicos de la zona, como el adobe (83.8\%), carrizo (7.5\%) y maguey (6.3\%).

\section{Sanidad}

Los ectoparásitos, en especial los piojos (conocidos en la zona como "piojo rojo" o "ugue”), fueron considerados como el principal problema sanitario (90.9\%). Diversos estudios confirman que los ectoparásitos son un problema frecuente en la crianza de cuyes (Vera y Vega, 1988; Kajjak et al., 1991; Chauca et al., 1994). Las enfermedades in- 
fecciosas y de alta mortalidad como salmonelosis y neumonía, denominadas comúnmente por los productores como "peste", fueron mencionadas por el $76.0 \%$ de los encuestados. Mayormente, no se realizaban medidas preventivas y no se empleaban medicinas. La práctica de tratamientos tradicionales a través de plantas medicinales estaba bastante difundida.

\section{Selección y empadre}

La selección de reproductores no era una práctica empleada, dado que el empadre era continuo y todos los machos tenían igual oportunidad de cubrir a las hembras. Los productores acostumbran sacrificar o vender a los cuyes más grandes. Es decir, en crianzas familiares se realiza una selección negativa (Chauca et al., 1994).

\section{Destete}

No se realizaba. Las crías se encontraban expuestas a aplastamientos y tenían que competir por el alimento con los más grandes.

\section{Destino de la producción}

Los cuyes presentaban un crecimiento lento, por lo que generalmente son consumidos o vendidos a partir de los 6 meses de edad, con un peso promedio de $966 \mathrm{~g}$. El destino de los cuyes era el autoconsumo y venta (71.2\%) o solo autoconsumo (28.8\%). La venta se realiza en el mercado de la ciudad de Santa Cruz, de preferencia los domingos, $o$ en la propia vivienda. Eran comercializados vivos a un precio promedio de diez nuevos soles (aprox. US\$ 3.40).

\section{Registros y controles}

Ninguno de los productores utiliza registros. Toda la información se recabó de forma verbal, siendo esto una de las limitantes del estudio. Es así, que esta encuesta sólo constituye un sondeo situacional, por lo tanto algunos elementos de la misma pueden estar influenciados por las apreciaciones personales de cada productor encuestado (FernándezBaca y Bojorquez, 1994).

Servicios

El 96.2\% de los encuestados manifestó no contar servicios de asistencia técnica, créditos financieros pecuarios, etc. El 67.5\% de las familias consideran que para mejorar la crianza de cuyes requieren de asistencia técnica, seguido de reproductores (12.5\%), pastos (8.8\%), e instalaciones (5.6\%), entre otros.

\section{ConClusiones}

- La crianza de cuyes en el distrito de Santa Cruz es conducida de manera tradicional, sobre la base de insumos y mano de obra disponibles en el hogar.

- El sistema de producción de cuyes identificado y caracterizado es de tipo familiar.

- Los factores limitantes identificados incluyen escasas técnicas de manejo, desconocimiento de la fisiología reproductiva de los cuyes, deficiente alimentación, instalaciones inadecuadas, carencia de planes sanitarios y pobres rendimientos productivos y reproductivos.

\section{Literatura Cittada}

1. Chauca L, Zaldívar M, Muscari J, Higaona R, Gamarra J, Florian A. 1994. Proyecto Sistemas de Producción de Cuyes. Tomo I. INIA-CIID. [Internet], [24 octubre 2008]. Disponible en: http://www.inia.gob.pe/documentos/ PSP\%20CUYES\%20Tomo\%201.pdf

2. Chauca L. 1997. Producción de cuyes (Cavia porcellus). Estudio FAO: Producción y sanidad animal-138. [Internet], [12 julio 2004]. Disponible en: http:// www.fao.org/DOCREP/W6562s/ W6562s00.htm 
3. Fernández-Baca E, Bojorquez C. 1994. Producción lechera en el valle del Mantaro: 1. Recursos disponibles para la producción. Rev Inv Pec, IVITA 7(1): 45-53.

4. INEI. 2004a. Banco de Información Distrital. Lima: INEI. [Internet], [15 junio 2004]. Disponible en: http:// www.inei.gob.pe

5. INEI. 2004b. III Censo Nacional Agropecuario 1994. Lima: INEI. [Internet], [09 junio 2004]. Disponible en: http://www.inei.gob.pe

6. Kajjak N. 1995. Experiencias de la crianza de cuyes en Arequipa y Huancayo. En: Serie Guía Didáctica: Crianza de Cuyes. Lima: INIA. p 118-124.

7. Kajjak N, Zaldívar M, Chauca L. 1991. Caracterización de sistemas prevalecientes de producción de cuyes. En:
Investigación en cuyes. Tomo I. INIA. [Internet], [10 octubre 2008]. Disponible en: http://www.inia.gob.pe/documentos/IF-Cuy.pdf

8. Ruíz M. 1989. El enfoque de sistemas en la investigación pecuaria y su metodología en América Latina. En: Ciencias Sociales y Enfoque de Sistemas Agropecuarios. Nolte E, Ruiz M. Lima: RISPAL. p 9-28.

9. Vera O, Vega D. 1988. Evaluación de la crianza de cuyes en los distritos de Huamanga y Huanta. En: Investigación en cuyes. Tomo I. INIA. [Internet], [10 octubre 2008]. Disponible en: http:// www.inia.gob.pe/documentos/IFCuy.pdf

10. Zaldívar R. 1989. Sistemas de producción amazónicos. Primer Informe. Pucallpa: IVITA. UNMSM. CIID. 80 p. 